\title{
Mechanics of Tubes Composed of Interlocking Building Blocks
}

\author{
Kyle Mahoney, Thomas Siegmund ${ }^{1}$ \\ School of Mechanical Engineering, Purdue University, West Lafayette, IN 47907, USA; \\ ${ }^{1}$ Corresponding Author, siegmund at purdue.edu
}

\begin{abstract}
Topologically interlocking material (TIM) systems are composed of convex polyhedral units placed such that building blocks restrict each other's movement. Here, TIM tubes are considered as rolled monolayers of such assemblies. The deformation response of these assembled tubes under diametrical loading is considered. This investigation employs experiments on additively manufactured physical realizations and finite element analysis with contact interactions. The internal load transfer in topologically interlocking tubes is rationalized through inspection of the distribution of minimum principal stress. A thrust-line (TL) model for the deformation of topologically interlocking tubes is established. The model approximates the deformation response of the assembled tubes as a the response of a collection of Mises trusses aligned with paths of maximum load transfer in the system. The predictions obtained with the TL-model are in good agreement with results of finite element models. Accounting for sliding between building blocks in the TL-model yields a predicted response more similar to experimental results with additively manufactured tubes.
\end{abstract}

Keywords: Architectured Material Systems, Topological Interlocking, Tube 


\section{Structures}

\section{Introduction}

Architectured material systems comprise of periodic geometric material features larger than the microstructure of the underlying material (e.g. a grain size) but smaller than the overall component of interest. Material architecture is of interest as a design approach as it can be used to tailor the response of structures to specific applications [1,2]. Synthetic architectures exist as lattice materials [3], kirigami or origami [4], and as assemblies of individual building blocks [5]. Many of these material architectures draw inspiration from natural occurrences of periodic geometry [6]. Plants and animals have evolved to possess skins, scales, armors, and other extremities that give enhanced functionality with structurally unique constitution $[7$, 8, 9]. Bamboo for example is composed of stacked tubular segments that each possess multi-cell micro-architecture [10], with the architecture seen as responsible for high compression and bending strength. Seed and nut shells were recently shown to possess a micro-architecture that results in high durability [11]. Seed shells composed of topologically interlocking 3D puzzle-type components were demonstrated to possess higher the strength that seed shells built from a fiber-type material. Epithelial cells in embryos have been found to take a scutoid shape during their growth, which allows the collection of cells to take on a tubular shape [12]. This collection of scutoid-shaped cells is much softer than the puzzle-type component assembly in nut shells, but still provides structure to a developing embryo. The driving mechanism in these examples of naturally resilient materials is a segmented 
and interlocking material architecture. It can thus be hypothesized that using this bioinspired principle, traditionally monolithic structures could be replaced with assemblies of interlocking building blocks to achieve a strong and resilient engineered structural response.

Topologically interlocking material (TIM) systems are one example of an architectured material system that utilizes the principle of material architecture through interlocking building blocks. Historically, interlocking building block systems have been used to construct floors and walls, see discussion in $[13,14]$. Recent efforts have revived these concepts in the context of a modern stone architecture for the 21st Century $[15,16,17,18,19,20]$. In the context of engineering material systems, the terminology Topologically Interlocking Material Systems has been introduced [5, 21, 22, 23, 24]. When considering an underlying brittle solid, these studies demonstrate an increase in toughness, or energy absorption capability, for the segmented system relative to the corresponding monolithic system. Even more significant, [25] demonstrated the simultaneous increase of strength and toughness for the segmented system over the corresponding monolithic one. Recent advances have focused on the altering of the mechanical properties of the TIM systems by manipulating the segmentation architecture. In [26] it was shown that heterogeneously segmented system do possess higher strength and toughness than those made of all identical building blocks. Furthermore, in [27] it was shown that the response of segmented systems depends strongly on the geometry of individual building blocks.

The unique load response of TIM systems is a result of the load transfer within the system, which differs from that in corresponding monolithic sys- 
tems. The periodic arrangement of building blocks and their interaction via contact allows load transfer to be described by the concept of force networks, and the deformation of TIM systems is then obtained by applying fundamental mechanics of material concepts to the deformation of the material in the force networks. While there exists a description of the mechanical response of planar TIM systems utilizing this force network concept [22, 28, 29], the behavior of non-planar TIM systems under external loading is not well defined. With the realization of a wide array of non-planar TIM geometries being plausible $[15,16,30,31,32]$, it is relevant that the mechanics of these structures be fully understood. Tunnels constructed of interlocking building blocks have been shown to have higher energy absorption capacity than standard construction methods [33]. Thermoacoustic instabilities in combustion chambers have also been mitigated with the energy absorption properties of TIM construction [34]. Further motivation is derived from the already mentioned study of interlocking seed wall structures [11].

The present study considers the mechanical behavior of TIM tubes under diametrical loading and investigates how tube aspect ratio and building block contact conditions influence the overall system response. Both an idealized system and a system conforming to experimental conditions is considered. Thereby, the mechanical response as predicted from FE-models can be compared to that of a closed form thrust line model and to a measured experimental response. 


\section{Methods}

\subsection{Material System Architecture}

TIM tubes are constructed from truncated quasi-tetrahedral building blocks. The plurality of building blocks is arranged to form a tubular structure. The discrete character of the TIM systems requires the presence of an external frame, or geometric constraint. These frames conform to the TIM tube at one of their axial surfaces. For the tubes, such frames constrain the collection of building blocks at each tube end. Each of the constraining frames is equivalent to one ring of fused-together building blocks. Figure 1 shows an example of a TIM tube configuration.

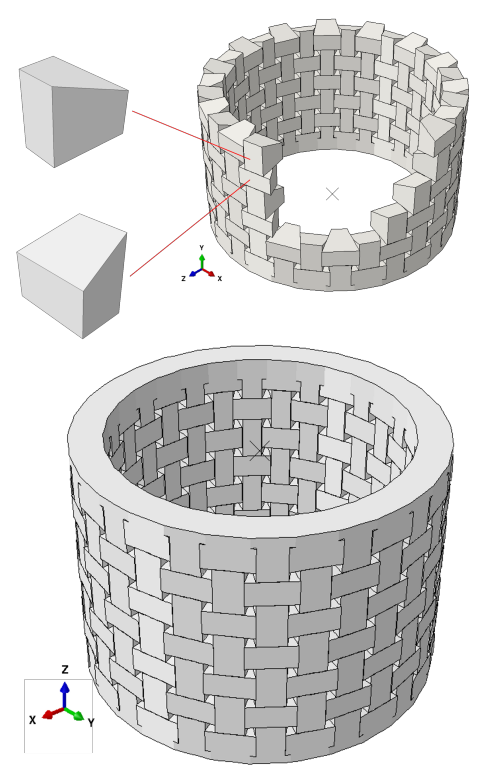

(a)

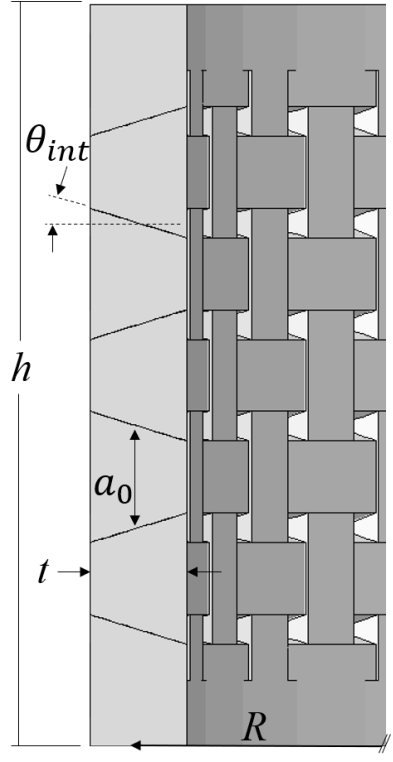

(b)

Figure 1: (a) Example of a TIM tube assembled by 150 individual building blocks and constrained by two rigid frames at the tube ends. (b) Detail of the axial cross-section of TIM tube. 
The building block geometry is defined by square prototiles of edge length, $a_{0}$, placed tangentially to a cylinder radius $R$. The square prototile edge length is related to the number, $n_{f}$, of building blocks (i.e. the number of facets) along the tube perimeter as $a_{0}=2 R \tan \left(\pi / n_{f}\right)$. Each edge of the square prototiles lies in its own plane oriented at the interlocking angle $\theta_{\text {int }}$ relative to a cylindrical coordinate system with its origin at the tubes center. Angles are defined from the $r-z$ plane for vertical edges and from the $r-\theta$ plane for horizontal edges. Alternating $\theta_{i n t}$ between its positive and its negative value when traversing the square prototile perimeter results in the intersections of the bounding planes being the vertices of quasi-tetrahedral building blocks. The resulting quasi-tetrahedra are of two distinct shapes as a result of the two possible orientations of planes relative to the reference cylinder surface.

All tetrahedral building blocks are truncated to a desired tube wall thickness, $t$. The number of building blocks along the $z$-axis, $n_{h}$, sets the tube height as $h=\left(n_{h}+2\right) a_{0}$. The tube aspect ratio is defined as $\varphi=h /(R+t)$. In the present study, three values of tube aspect ratios are considered, $\varphi=1.6$, 2.3, and 3.0. TIM tubes are constructed with an interlock angle of $\theta_{\text {int }}=17^{\circ}$. Tube structures built as an arrangement of non-interlocking building blocks, $\theta_{\text {int }}=0^{\circ}$, are considered for comparison.

\subsection{Experimental Methods}

Physical realizations of TIM tubes were created by the Polyjet additive manufacturing (AM) processes using an Objet350 Connex (Stratasys Ltd.) 3D printer. As-manufactured TIM tubes undergo diametral loading by a flat

punch, Fig. 2. The flat punch is positioned at the mid-height of the TIM 


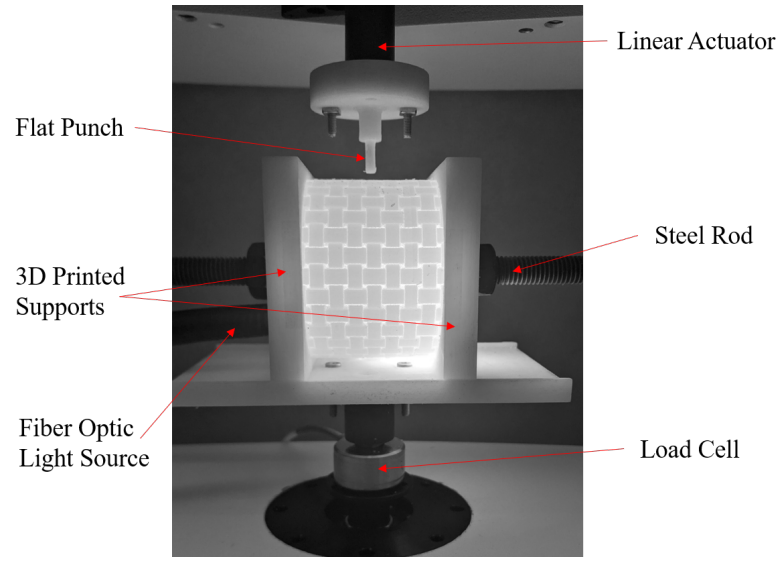

(a)

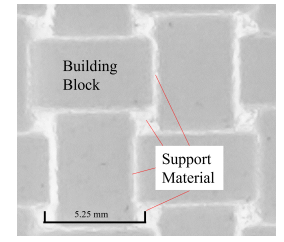

(b)

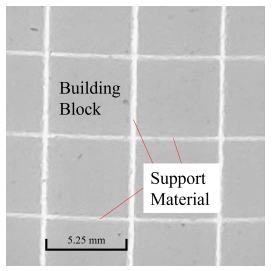

(c)

Figure 2: (a) Experimental setup for diametrical loading experiments of TIM tubes by a flat punch. A fiber light is inserted into the tube such that the back illumination enables the visualization of the TIM system architecture. Detailed view of (b) interlocking building blocks $\left(\theta_{\text {int }}=17^{\circ}\right)$ and (c) non-interlocking building blocks $\left(\theta_{\text {int }}=0^{\circ}\right)$ surrounded by support material.

tube and initially sits flush with the outer face. The load response of TIM tubes under quasi-static applied displacement conditions captured using a BOSE ElectroForce 3200 mechanical test instrument.

The TIM tubes are printed as integrated assemblies of all building blocks and end frames in a PolyJet (tm) print process. Thereby, building blocks (VeroWhite (tm), Stratasys Ltd.) were separated by a layer (0.3 mm thickness) of of the PolyJet support material FullCure705 (Stratasys Ltd.). Building blocks were separated by a layer ( $0.3 \mathrm{~mm}$ thickness) of of the Polyjet support material FullCure705 (Stratasys Ltd.). The support material was removed from the TIM tube external surface but left in place between the building blocks. 
In order to realize a system where the support material (mortar) provides minimal mechanical strength, the support material is embrittled. The FullCure support material in an as-printed state is already significantly more compliant and weaker than the VeroWhite material used for the building blocks. In addition, aging through exposure to UV light degrades the strength of the support material [35]. Specimens were aged for seven days before the mechanical loading experiments. The resulting TIM tube systems then are dominated by the geometry of the building blocks, their mechanical behavior, and interactions rather than by the support material filling the gap.

The end frames of the TIM tubes were connected to one another by use of a threaded steel rod. Nuts and washers are threaded onto each end of the rod and tightened to near zero pressure by monitoring the pressure through thin film pressure sensors. Thereby, the TIM tubes is axially constrained with zero pre-load. The stiffness of the steel rod connecting frames is approximately $20 \mathrm{E} 6$ times that of the elastic connectors considered in the finite element models. Thus, the resulting conditions are such that the ends of the tubes are essentially fixed in space. For visualization purposes a glass fiber light tube is inserted through the end plates to provide back-light illumination.

\subsection{Finite Element Models}

TIM tubes are constructed from linear elastic solids with elastic modulus $E$ and Poisson's ratio $\nu$. Building blocks interact with each other through contact and friction. TIM tubes are loaded diametrically by a flat punch. Thereby, one of the building block located at the mid-height position of the TIM tube is loaded with the end face of a cylinder $\left(d<a_{0}\right)$ whose axis is aligned with the $r$-coordinate, Figs $3 \mathrm{a}$ and $3 \mathrm{~b}$. 


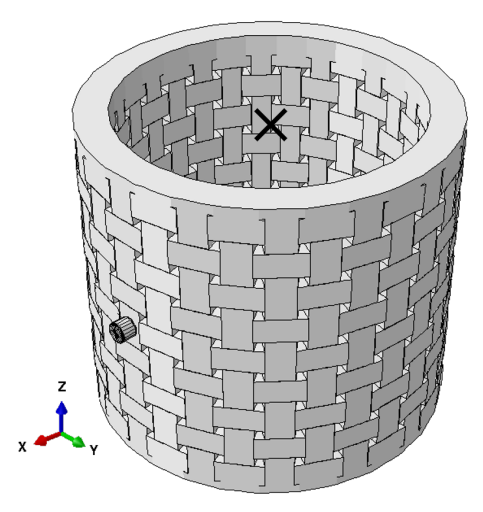

(a)

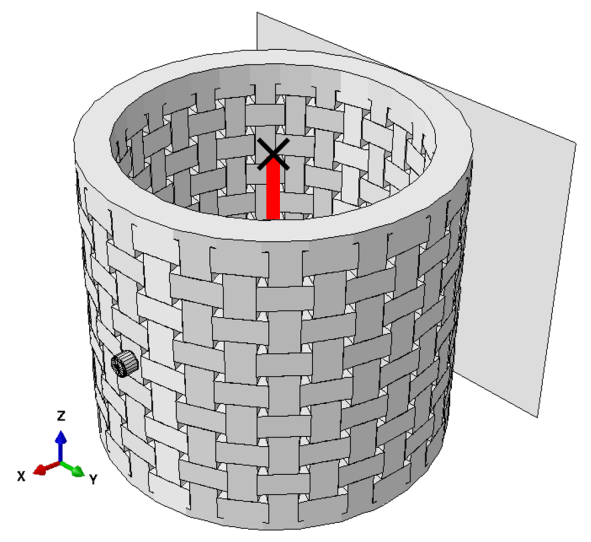

(b)

Figure 3: TIM tubes and loading conditions: (a) Loading via a flat punch and fixed frame boundary condition (FP) and (b) loading via a flat punch and frames connected via an elastic element (shown in red), the system rests on a flat surface (FP-E).

TIM building blocks were assigned the following mechanical properties, to replicate the polymer used in experiments: $E=1827 \mathrm{MPa}$ and $\nu=0.35$. Both the frames and the load-inducing punch were considered as rigid bodies. A density value of $\rho=950 \mathrm{~kg} / \mathrm{m}^{3}$ was used. A mass scaling factor of 100 was employed to decrease computational cost and suppress the kinetic energy. Two sets of contact conditions are considered in the FE simulations. In an idealized system condition, the contact stiffness is high and approaches a hard contact. In that case, contact between all building blocks in the system was characterized by a linear pressure-overclosure formulation with stiffness 50 times that of the finite elements, $K_{c}=63.95 \mathrm{kN} / \mathrm{mm}$. Friction is described by a Coulomb friction model. The coefficient of friction considered was $\mu=0.6$, as this is the upper bound of $\mu$ for dry materials. In FE-models seeking to approximate the experimental system, the contact and friction interaction 
can depart significantly from the idealized conditions. The actual value of the contact stiffness is then determined through a fit to the experimental data. Furthermore, an extended Coulomb friction model with a shear stress cut-off $\tau_{0}$ is employed. The actual parameter value of the contact stiffness and $\tau_{0}$ are determined through a fit to the experimental data.

Two types of constraint configurations are considered. First, the constraining frames are considered as rigid and spatially fixed, Fig. 3a. Such a boundary condition (FP) resembles a set-up where the TIM tube serves as a connector between two stiff, and strongly connected components. Alternatively, rigid frames are constrained to one another by an elastic element with its ends positioned at the centroids of the two opposing frames. In such a configuration (FP-E), the TIM tube is self-constrained and can be handled as an independent part. During loading, the tube is considered to be resting on a flat, rigid surface, Fig. 3b. The stiffness $K_{s}$ of the elastic connection between the two frames is an additional system parameter. Here, it was considered as equal to the axial stiffness of the equivalent monolithic tube $K_{M}=E A_{M} / h$, where $A_{M}=(R+t / 2)^{2} \pi-(R-t / 2)^{2} \pi$. Consequently, $K_{s}=59.7 \mathrm{kN} / \mathrm{mm}$ for $\varphi=1.6, K_{s}=41.5 \mathrm{kN} / \mathrm{mm}$ for $\varphi=2.3$, and $K_{s}=31.8$ $\mathrm{kN} / \mathrm{mm}$ for $\varphi=3.0$.

FE-models of the TIM tube systems are solved for displacement-controlled loading by a flat punch. Computations are executed by use of an explicit FE algorithm (ABAQUS Explicit) but only the quasi-static response is of concern. In addition to the force-displacement response of the punch, computed values of external work $W_{e x}$, strain energy $U_{e l}$, frictional dissipation $U_{f}$ and contact penalty work $U_{c}$ were recorded. Other system energy components 
(viscous dissipation, artificial energy, and kinetic energy) were negligible before total system failure occurred. In idealize model configurations, only $W_{e x}$, $U_{e l}$ and $U_{f}$ are of concern and $U_{c}$ is negligible. In model configurations representing the experiments, only only $W_{e x}, U_{c}$ and $U_{f}$. In that case $U_{e l}$, the strain energy, is insignificant.

TIM building blocks and monolithic tubes were discretized with linear, hexahedral, reduced integration elements. Enhanced hourglass control was used with the reduced integration elements. The mesh convergence of the simulations was investigated by monitoring maximum reaction force $\left(F_{\max }\right)$ values with mesh densities ranging from 5 to 20 seeds per building block unit edge. At 15 seeds per polyhedra edge the difference in $F_{\max }$ between seed sizes was less than $1 \%$, so building blocks in all computations were meshed with 15 seeds per edge. With this value, individual TIM building blocks were comprised of 3600 elements (type C3D8RH of the ABAQUS/Explicit code). For details of the convergence study, see Appendix A.

\section{Experimental Results}

Figure 4 depicts measured force-displacement data for both the TIM tubes and the non-interlocking tubes. The response of the TIM tubes $\left(\theta_{\text {int }}=17^{\circ}\right)$ features an initial elastic regime followed by a sharp drop in measured force, Fig. 4a. The stiffness of TIM tubes decreases with tube aspect ratio. One observes a rather abrupt load drop past a peak load value. Final failure occurs at displacements approximately equal to the tube wall thickness. Non-interlocking tubes $\left(\theta_{\text {int }}=0^{\circ}\right)$ exhibit stiffness and strength that are independent of tube aspect ratio, Fig. 4b. A sharp drop in measured force 
is still observed past the peak load, however. Using elementary mechanics of materials concepts, the strength of support material is computed to be $\tau_{0}=F_{\max } /\left(4 a_{0} t\right)=0.08 \mathrm{MPa}$.

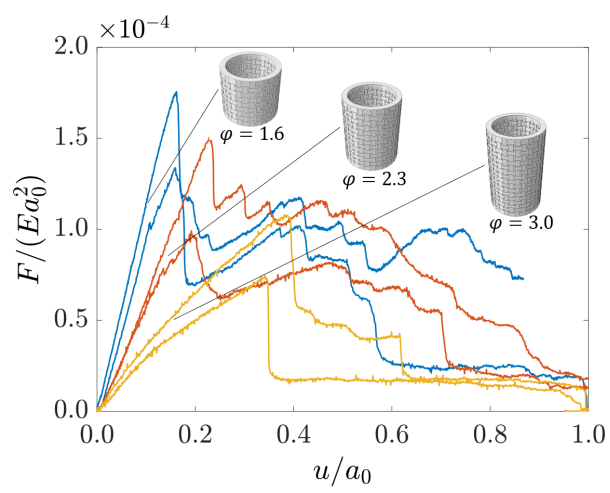

(a)

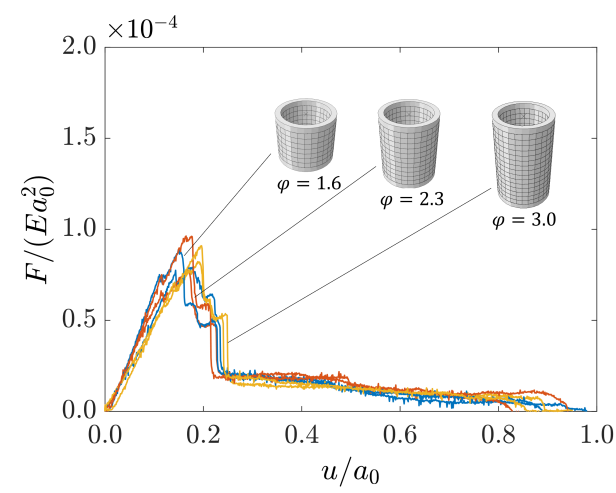

(b)

Figure 4: Normalized data of the measured force-displacement response for: (a) TIM tubes $\left(\theta_{i n t}=17^{\circ}\right)-\mathrm{FP}$ and $(\mathrm{b})$ non-interlocking tubes $\left(\theta_{i n t}=0^{\circ}\right)-\mathrm{FP}$.

Details of the deformation and failure mechanism can be deduced from Fig. 5. In the experiments, one observes that the entire TIM tube face is deflected initially, Fig. 5a. The loaded building block is pushed in by the punch after support material between blocks begins to fail, Fig. 5b. If pushout was the only active deformation mechanism, then only this building block would incur significant displacement. This is however, not the case in the topologically interlocked configuration. Figures $5 \mathrm{~b}$ and $5 \mathrm{c}$ demonstrate that the deformation of the TIM tube systems affects not only the building block in contact with the punch. In Fig. 5b adjacent building blocks are found to be tilted from their initial position, and in Fig. 5c building blocks even further removed from the loaded block are not only tilted but also shifted radially outward from their initial position. After the push-out of the center 


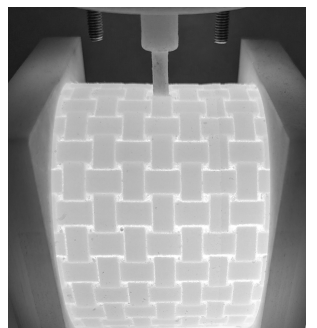

(a)

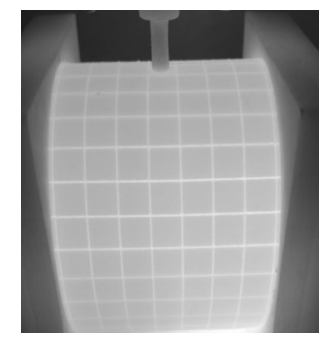

(e)

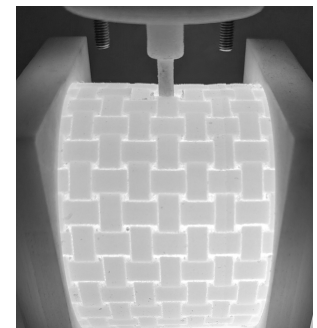

(b)

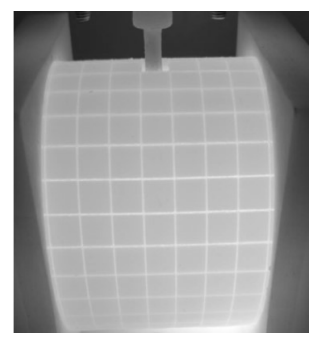

(f)

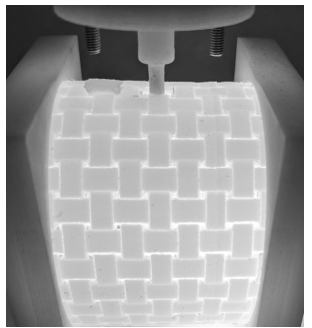

(c)

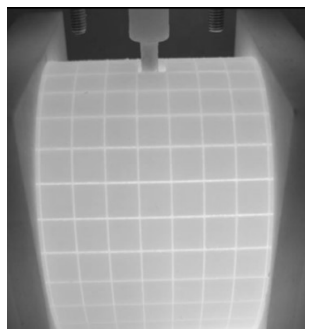

(g)

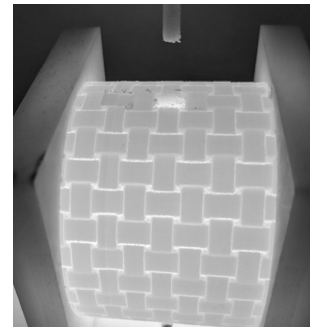

(d)

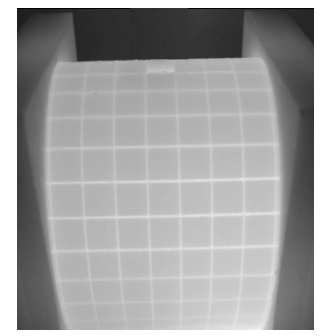

(h)

Figure 5: Snapshots of deformed TIM and non-interlocking $\left(\theta_{\text {int }}=0^{\circ}\right)$ tubes $(\varphi=1.6)$ during loading experiment. (a-d) TIM tube $\left(\theta_{i n t}=17^{\circ}\right.$ ) - FP: (a) $u / a_{0}<0.2$, (b) $u / a_{0}=0.5$, (c) $u / a_{0}=1.0$ and (d) removed center block. (e-h) Non-interlocking tube $\left(\theta_{\text {int }}=17^{\circ}\right)$ FP: (e) $u / a_{0}<0.2$, (f) $u / a_{0}=0.5$, (g) $u / a_{0}=1.0$ and (h) removed center block.

block at final stages of deformation, the TIM tube structure remains intact minus this building block. The deformation and failure of non-interlocking tubes on the other hand is entirely due to the push-out of the loaded building block. Before support material failure the entire tube face is deflected like in TIM tubes, Fig. 5e. Afterwards, the loaded building block is pushed in while the positioning of surrounding blocks remains unchanged, Fig. $5 \mathrm{f}$ and 5g. The non-interlocking tube remains intact minus the pushed-out building block after failure, Fig. 5h. 


\section{Analysis}

\subsection{Idealized Models}

Figure 6 a depicts the computed force-displacement response of TIM tubes with fixed frame boundary conditions and flat punch loading. The response of the TIM tube is skew-parabolic and displays gradual failure beyond the maximum load. TIM tubes are found to fail at an applied displacement 1.3 to 1.7 times the tube thickness. Figure $6 \mathrm{~b}$ documents the computed forcedisplacement response of TIM tubes with elastically constrained frames and flat punch loading. The shape of the response and flat punch loading is preserved in case of the model with elastically connected frames; force levels are lower but the applied displacement to failure is slightly higher.

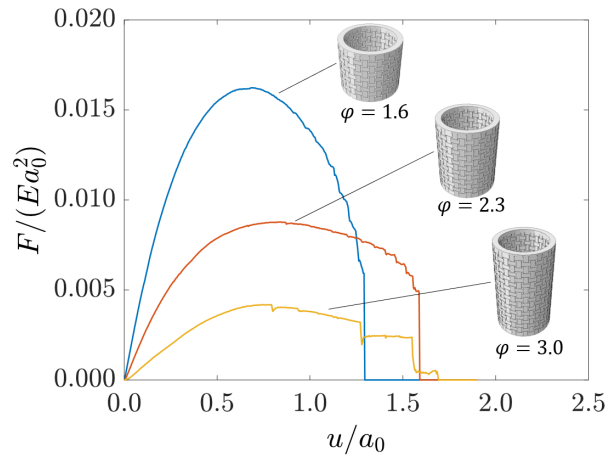

(a)

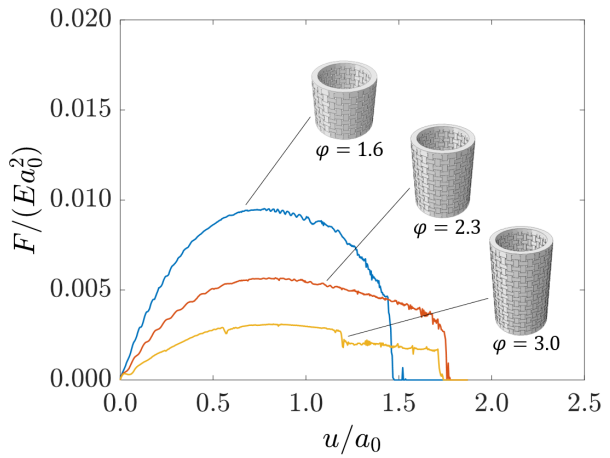

(b)

Figure 6: Normalized force-displacement response for: (a) TIM tubes $\left(\theta_{i n t}=17^{\circ}\right)$ - FP and (b) TIM tubes $\left(\theta_{i n t}=17^{\circ}\right)$ - FP-E.

TIM tube internal load transfer is visualized as contour plots of the minimum principal stress, $\sigma_{p, m i n}$, in Fig. 7. Load transfer within TIM systems is dominated by compressive stresses, with tensile loads restricted to the frames, 
and the connection between frames (if present). This is distinct from the load transfer in a monolithic structure, where material is loaded by bending under transverse indentation and a distribution of tensile and compressive stresses exists. Distributions of $\sigma_{p, \min }$ on axial sections of the TIM tubes were created at the instance of the force maximum, and at force levels of half the force maximum pre- and post-peak.

These paths of load transfer can be considered thrust-lines forming a force network within the system. The thrust-lines are initially oriented diagonally along the exposed cross-section such that they originate underneath the punch and terminate at the binding frames, Fig. 7a. The evolution of thrust-lines is a result of the displacement of the center building block as well as the rotation of neighboring building blocks. When the system strength is reached the thrust-lines in this cross-section are oriented with the tube axis, Fig. 7b. These thrust-lines are observed to remain present even at displacements larger than the wall thickness of the TIM tube, Fig. 7c. Due to the slip of the center block relative to its neighbors, the shape of the thrust-line is modified and now straddles the inner tube surface rather than extending from the tube exterior to the interior. The load transfer is not limited to only a few building blocks. The domain of the tube under stress spreads from the loaded building block to many points along the tube perimeter. Other thrust-lines composing the over-arching force network are evident from the visible stress contours outside of the cut cross-section. These thrust-lines also originate underneath the punch, but terminate at different points along the frames' perimeter. Force networks remain intact up until some point at which the center building block is punched out from the tube. This observation was 
consistent for all aspect ratios considered.

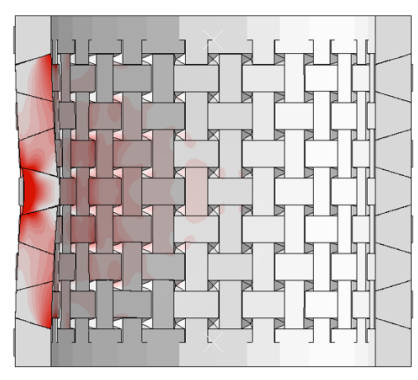

(a)

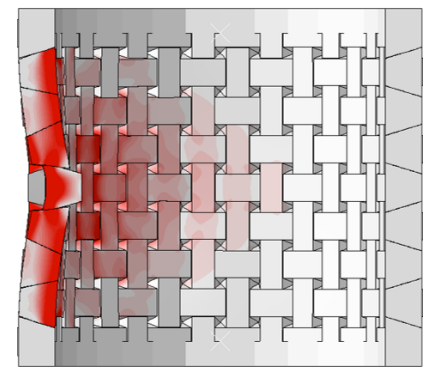

(b)

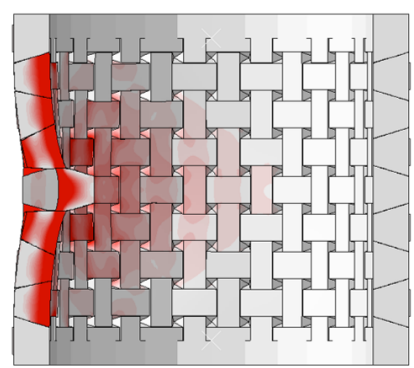

(c)

Figure 7: Contours of the computed minimum principal stress, $\sigma_{p, \text { min }}$, from FE-models representing the idealized configuration of a TIM tube $\left(\theta_{\text {int }}=17^{\circ}, \varphi=1.6\right)$ - FP: (a) $u / a_{0}=0.25$, (b) $u / a_{0}=0.75$, and (c) $u / a_{0}=1.25$. Contour scale: $[0$ (grey), -50 (red)] MPa.

The computed system energies for $\theta_{i n t}=17^{\circ}$ are depicted in Fig. 8. At initial stages of deformation, the elastic strain energy dominates over the frictional dissipation. As deformation progresses, strain energy stored is reduced in magnitude and frictional dissipation becomes dominant. The tube aspect ratio determines if slip or elastic deformation dominates the system deformation. For the smallest aspect ratio, deformation to about the peak load is dominated by elastic energy. For the two cases with higher aspect ratio, deformation far into the softening regime remain dominated by elastic energy. In the highest aspect ratio case, $\varphi=3.0$, essentially the entire deformation is dominated by elastic deformation and slip only becomes relevant during the final stages of loading.

The thrust-line (TL) model approach of $[22,28,29]$ is followed to establish an analytical model for the calculation of the force-displacement response. Thrust-lines are domains within the system of high values of compressive 


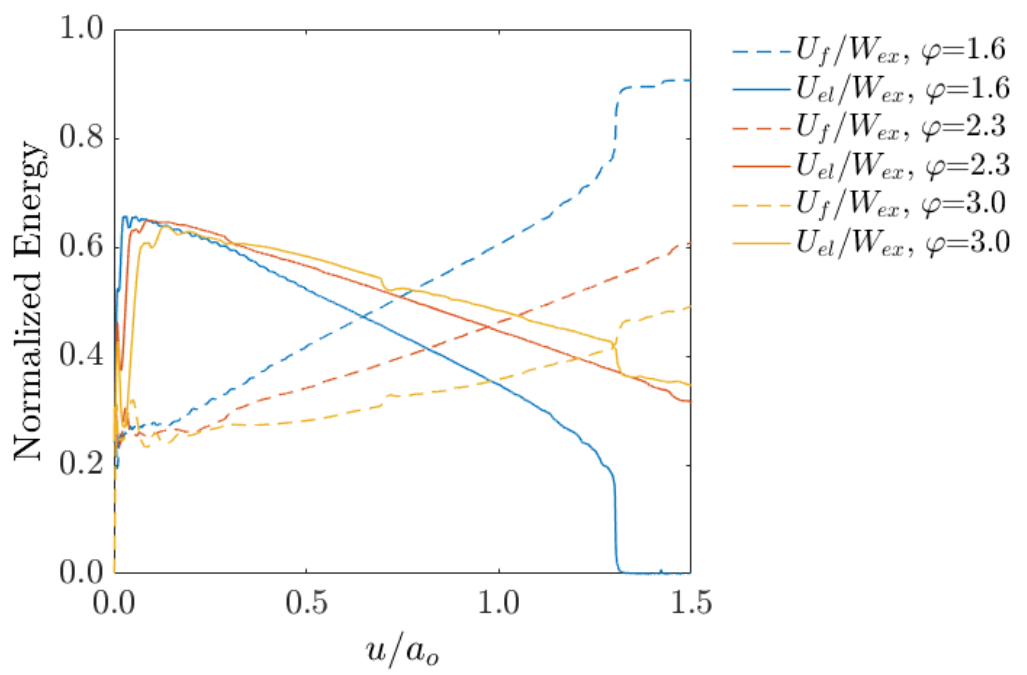

Figure 8: Ratios of computed model energies $U_{e l} / W_{e x}$ and $U_{f} / W_{e x}$ for the idealized model configuration of TIM tubes $\left(\theta_{\text {int }}=17^{\circ}\right)$ - FP.

principal stress, Fig. 7. In the model, thrust-lines are represented as trusses. Pairs of such trusses form a collection of Mises trusses. The mechanical analysis of Mises trusses is then used to establish equations to predict the load response. Each truss is assigned a local coordinate system such that all truss coordinate systems share a $y$-axis. The reactions $F_{x i}$ (thrust force) and $F_{y i}$ (shear force) at the abutments (the rigid end frames) of each of the Mises trusses $(i)$ are computed as:

$$
\begin{aligned}
& F_{x i}\left(\eta_{i}\right)=E A_{i}\left[1-\frac{\cos \left(\eta_{0 i}\right)}{\cos \left(\eta_{0 i}-\eta_{i}\right)}\right] \cos \left(\eta_{0 i}-\eta_{i}\right) \\
& F_{y i}\left(\eta_{i}\right)=E A_{i}\left[1-\frac{\cos \left(\eta_{0 i}\right)}{\cos \left(\eta_{0 i}-\eta_{i}\right)}\right] \sin \left(\eta_{0 i}-\eta_{i}\right)
\end{aligned}
$$

Here, the change in angle of inclination of the truss $i$ is $\eta_{i}=\eta_{0 i}-\tan ^{-1}\left(2 h_{i} / L_{i}\right)$ with $h_{i}=h_{0 i}-u$. The load response of each Mises truss relates to the initial 
truss height $\left(h_{0 i}\right)$, the truss span $\left(L_{i}\right)$, constitution $(E)$, and an equivalent cross-section area $\left(A_{i}\right)$. The elastic modulus of the trusses is assumed to be that of the VeroWhite (tm) polymer used in realizations of TIM tubes. Then, the truss section area value was estimated by fitting the response calculated with the TL-model to that from the FE-model. The net reaction force for each Mises truss is: $F_{i}=2 F_{y i}$.

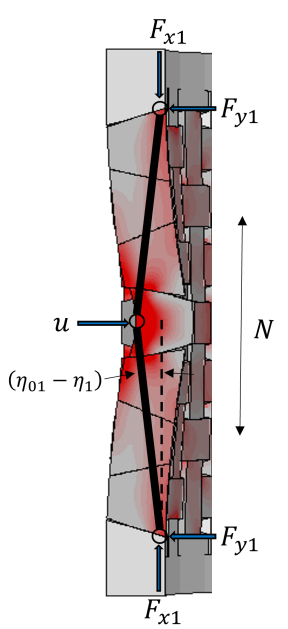

(a)

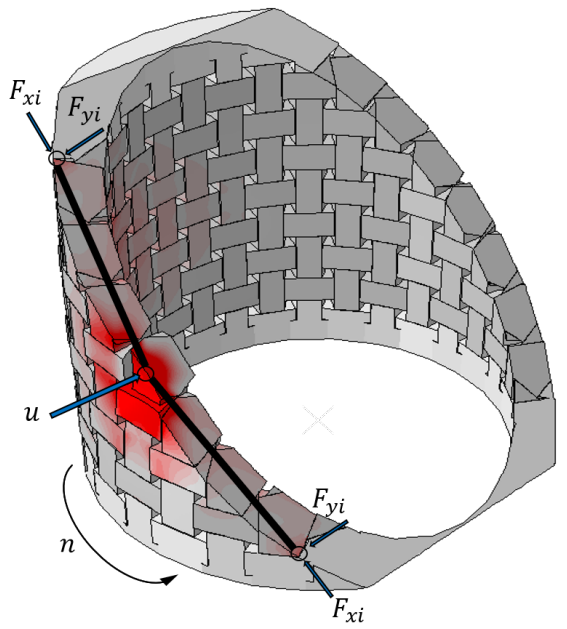

(b)

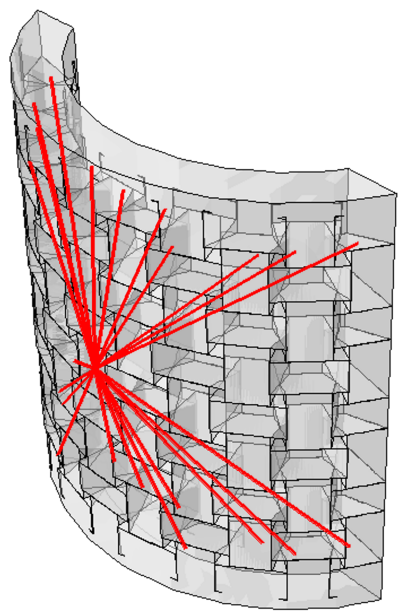

(c)

Figure 9: Deformed TIM tube geometry $(\varphi=1.6)$ and $\sigma_{p, \min }$ contours on (a) an axial section with Mises truss $F_{1}$ overlaid and (b) an oblique section with an off-axis Mises truss overlaid. In the depicted instance $i=4$. The circumferential distance between abutments of $F_{1}$ and $F_{i}$ is $(i-1) a_{0}$. Contour scale: [0 (grey), -100 (red)] MPa. (c) All Mises trusses (red) for the present model geometry are overlaid on translucent TIM tube geometry.

Mises trusses are arranged to align with thrust-lines observed in minimum principal stress contours from FE-models, Fig. 9. All truss apexes intersect on the face of the loaded building block at the point of punch contact. Truss members span from this point to discrete abutments along the rigid end 
frames. Truss 1 is aligned with the TIM tube axis, Fig. 9a. The abutment of a truss $(i>1)$ is placed at the tube end such that the circumferential distance from the abutment of truss 1 is $(i-1) a_{0}$. The oblique trusses $(i>1)$ then span the tube diagonally, Fig. 9b, and are symmetric about the tube mid-plane. This leads to a pyramidal arrangement of Mises trusses with the loaded building block at the apex of the pyramid, Fig. 9c. A truss $(i)$ must be entirely within the tube wall thickness, thus satisfying the condition:

$$
1-\cos \left[\frac{\pi(i-1)}{n}\right]<\frac{t}{R}, \quad i=1, n .
$$

The number of Mises trusses needed to be considered for a given $n$ is $(2 p-1)$, where $p$ is the largest integer $i$ satisfying the condition in eqn. 3 . The total load response of the TIM tube is then equivalent to the sum of the reactions of Mises trusses, eqn. 4.

$$
F=F_{1}+2 \sum_{i=2}^{p} F_{i} .
$$

There are two types of support configurations for the Mises trusses. One group of trusses has a termination at the end frames at the tube's inner surface, Fig. 9a. The second group of trusses has a termination at the end frames at the tube's outer surface, Fig. 9b. A truss $i$ is in the first group if its span is entirely within the tube wall thickness (in this configuration) by satisfying the condition in eqn. 5 . Otherwise the truss $i$ is in the second group. For the tubes considered here, trusses 1 and 2 are in the first group, and trusses $3,4,5$, and 6 are in the second group. 


$$
\frac{1}{4} \sqrt{\frac{\left[4 R^{2}-t^{2}\right]^{2}[\cos (2 i \phi)-1]}{4 R^{2} \cos (i \phi)-t^{2} \cos (i \phi)-4 R^{2}-t^{2}}} \geq R-\frac{t}{2}
$$

Consequently, there are two equations for the calculation of the initial angle of inclination of the Mises trusses with respect to the local truss span, $\eta_{0 i}$. The number of Mises trusses in the first group for a given $n$ is $(2 q-1)$, where $q$ is the largest integer $i$ satisfying the condition in eqn. 5 . The number of Mises trusses in the second group is then $(2 p-1)-(2 q-1)$. This is described in terms of axial building block count $N$, unit size $a_{0}$, tube radius $R$, and facet angle $\phi=2 \pi / n$ in eqns. 6 and 7 .

$$
\begin{gathered}
\tan \left(\eta_{0 i}\right)=\frac{2 R\{1-\cos [(i-1) \phi]\}+t\{1+\cos [(i-1) \phi]\}}{\sqrt{a_{0}^{2} N^{2}+4 R^{2} \sin ^{2}[(i-1) \phi]}}, \quad i<q \\
\tan \left(\eta_{0 i}\right)=\frac{(2 R+t)\{1-\cos [(i-1) \phi]\}}{\sqrt{a_{0}^{2} N^{2}+4 R^{2} \sin ^{2}[(i-1) \phi]}}, \quad i \geq q
\end{gathered}
$$

Figure 10 shows the force-displacement responses of TIM tubes as computed with the TL-model. For comparison, results obtained with the FEmodels of identically sized TIM tubes are shown (fixed frames, flat punch loading). The TL-model is fitted to the results from the FE-model by matching the initial stiffness of the TIM tube with $\varphi=1.6$. We consider $A_{i}=$ const., and a value of $E A_{i}=29,597 \mathrm{~N}$ in the TL-model is found to best replicate the results of the respective FE-model. The TL-model then used is used for the analysis of TIM tubes with $\varphi=2.3$ and $\varphi=3.0$. For all aspect ratios, the TLM overall predicts the initial stiffness and strength well. The load decay past the peak force is more pronounced in the data from FE-models, and the TL-model consistently predicts significantly larger dis- 
placements to failure. This finding is attributed to sliding between building blocks in FE-models which is not accounted for in the TL-model.

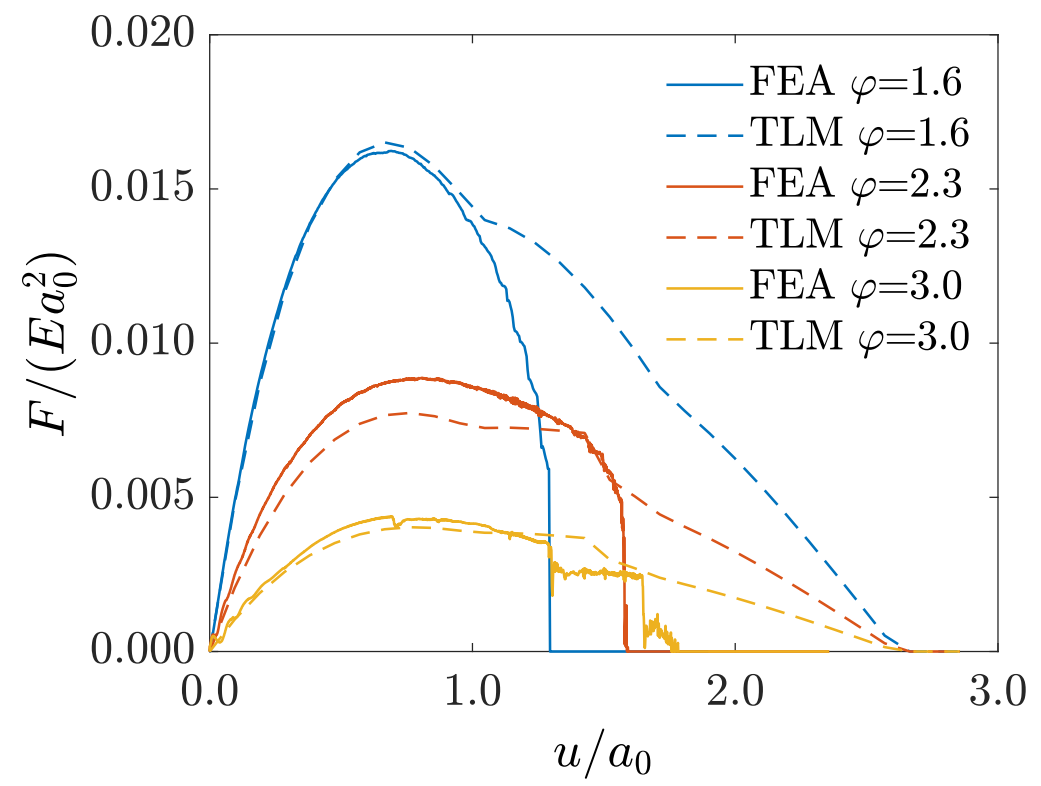

Figure 10: The force-displacement response as computed with the FE-model and the thrust-line model (TLM) for TIM tubes with $\varphi=1.6,2.3$, and 3.0.

\subsection{Realistic Models}

A comparison of the predicted TIM tube response for the idealized system and the respective physical realization reveals significant difference. Both the FE-model and the TL-model thus require modifications from their original form.

It is found that a decrease in the contact stiffness and the activation of a friction limit in FE-models leads to a response approximating the experimental results of the TIM tubes, Fig. 11. First, the contact stiffness is lowered to approximate the initial stiffness of the TIM tubes. A contact stiffness 
value of $K_{c} / 1250$ was found to provide a good match to all experimental data. Subsequently, the frictional shear stress limit is set to the strength of support material determined in experiments, $\tau_{0}=0.08 \mathrm{MPa}$. With these model parameters, the FE-model predicts stiffness and strength to again decline with increasing tube aspect ratio, Fig. 11. The failure of the TIM tubes is now predicted to occur at much reduced magnitudes of applied displacements. The FE-model predicts the presence of significant load drops past the peak load, which is also in agreement with the experimental observation. However, the FE-models predict the load drops to occur a later stages of loading than observed in the experiments.

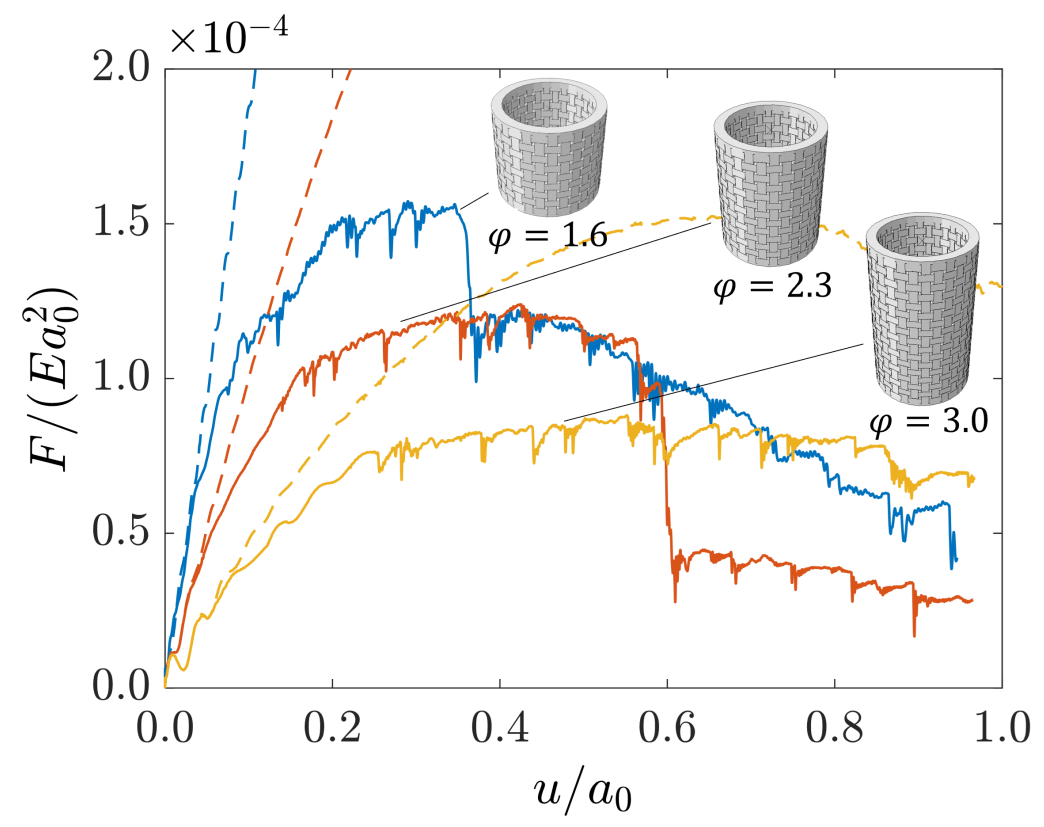

Figure 11: Normalized force-displacement response as obtained from FE-analysis with parameter estimates for experimentally measured response: TIM tubes $\left(\theta_{i n t}=17^{\circ}\right)$ - FP.

Computed distributions of $\sigma_{p, \min }$ on axial sections of the TIM tubes again 
were are depicted at the instance of the force maximum, and at force levels of half the force maximum pre- and post-peak. The shape of the force network as obtained from the the FE-model for the experimental system is initially similar to the one in the FE-model of the idealized system, Fig. 12a vs. Fig. 7a. Deviations from the idealized model occur at larger displacements due to the increased amount sliding between building blocks. In addition, while the center building block being displaced radially inward, other building blocks in the TIM tube are displaced radially outward, Fig. 12b and 12c. This leads to an earlier interruption of thust-lines within the TIM tube and a smaller displacement to failure.

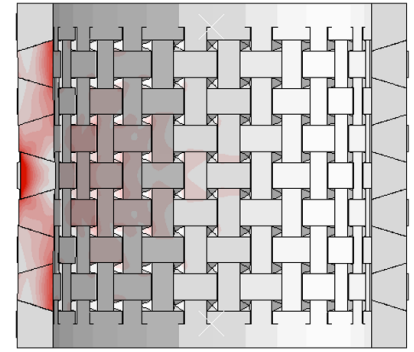

(a)

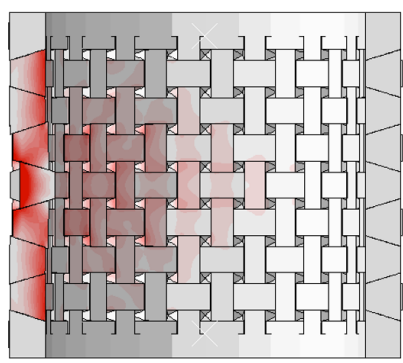

(b)

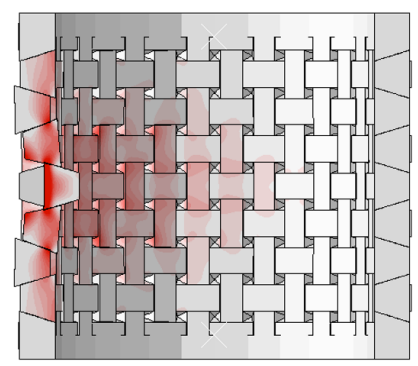

(c)

Figure 12: Contours of the computed minimum principal stress, $\sigma_{p, \text { min }}$, in from FE-models representing the experiments. (a-c) TIM tube $\left(\theta_{i n t}=17^{\circ}, \varphi=1.6\right)$ - FP: (a) $u / a_{0}=0.1$, (b) $u / a_{0}=0.3$, and (c) $u / a_{0}=0.7$. Contour scale: $[0$ (grey), -1 (red) $]$ MPa.

The computed system energies for $\theta_{i n t}=17^{\circ}$ are depicted in Fig. 13. With the realistic contact conditions, frictional dissipation becomes dominant in the system energy much earlier compared to idealized configurations. Now, slip dominates the system deformation before the peak load is reached for all aspect ratios. The ratio of frictional dissipation to stored elastic energy is also higher than in idealized configurations. Again, the dominance of sliding 
occurs at later stages of loading for higher aspect ratio TIM tubes.

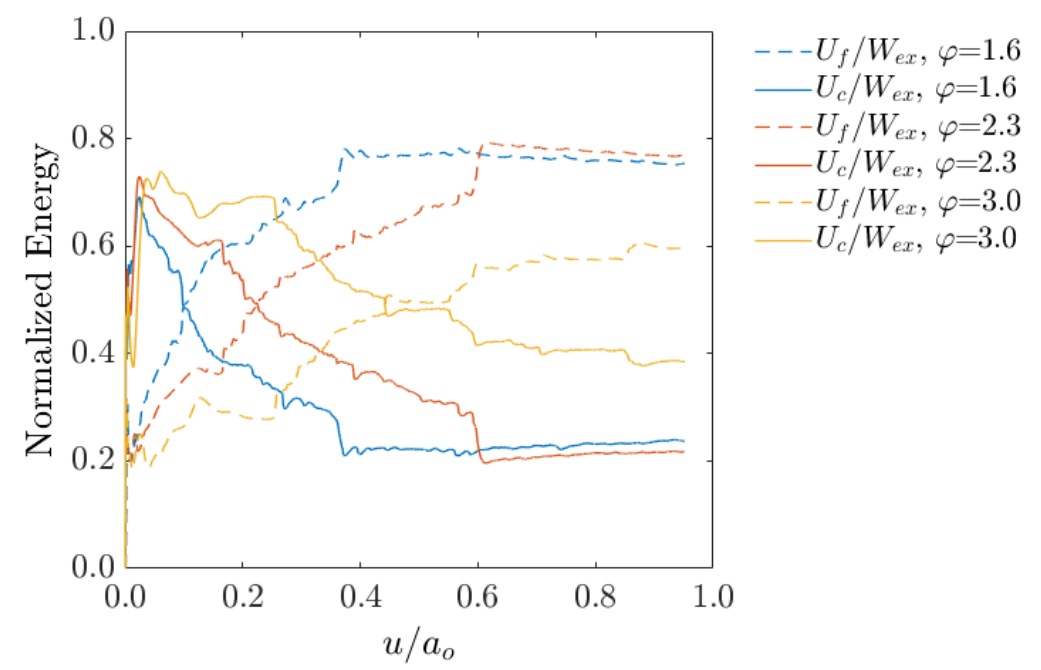

Figure 13: Ratios of computed model energies $U_{c} / W_{e x}$ and $U_{f} / W_{e x}$ for the model configuration representing the experimental system: TIM tubes $\left(\theta_{i n t}=17^{\circ}\right)$ - FP.

The TL-model for TIM tube behavior must be modified to account for sliding between building blocks. This is accomplished by allowing some fraction of applied displacement increments to account for sliding. This configuration is implemented as a modification of the reference configuration for the Mises truss. Thereby, the initial angle of a truss, $\eta_{0 i}$, is decreased by a fraction of the angle change $\eta_{i}$ due to the applied displacement. The fraction of angle change to be assumed as slip is referred to as the slip factor, $S_{f}$. The onset of slip is determined with a individual truss force criterion. Slip will not be introduced to an individual truss unless the shear component of the truss reaction exceeds a critical force, $F_{c}$. As suggested in [36], the critical force is related to interface frictional strength such that $F_{c}=\tau_{0} A_{c}$ where $\tau_{0}$ is the joint shear strength and $A_{c}$ is the contact area between building blocks. 
Deformation due to slip in TIM tubes is then described within the TL-model by eqn. 8 .

$$
\eta_{0 i}= \begin{cases}\eta_{0 i}, & \text { if } F_{i}<F_{c} \\ \eta_{0 i}-S_{f} \eta_{i}, & \text { if } F_{i} \geq F_{c}\end{cases}
$$

The predictions obtained with the slip-inclusive TL-model are presented in Fig. 14. The parameter values of TL-model are again estimated to match the stiffness of the TIM tube with $\varphi=1.6$, i.e. $E A_{i}=$ const. $=180 \mathrm{~N}$. From a comparison to experimental data and FE-results, it can be seen that the TL-model approximates the initial stiffness of the TIM tubes well. A critical force value of $F_{c}=1.0 \mathrm{~N}$ results in the model-predicted strength matching experiment results. The decay of indentation force with additively manufactured TIM tubes is best replicated with a slip factor of $S_{f}=0.8$. All of the above values are used in the TL-model with dimensions of TIM tubes with $\varphi=2.3$ and 3.0. The additively manufactured TIM tube stiffness and strength are reasonably approximated for each aspect ratio.

\section{Discussion}

We define the geometry of TIM tubes as consisting of two types of quasitetrahedral building blocks. This is in contrast to the interlocking configuration emerging from a planar grid of prototiles. In that case only one uniquely shaped building block is needed, yet defining interlocking geometry from a quasi-cylindrical grid of prototiles introduces two complementary polyhedra. TIM tubes were realized by a PolyJet (tm) additive manufacturing process

which creates a material architecture that is functionally similar to those of 


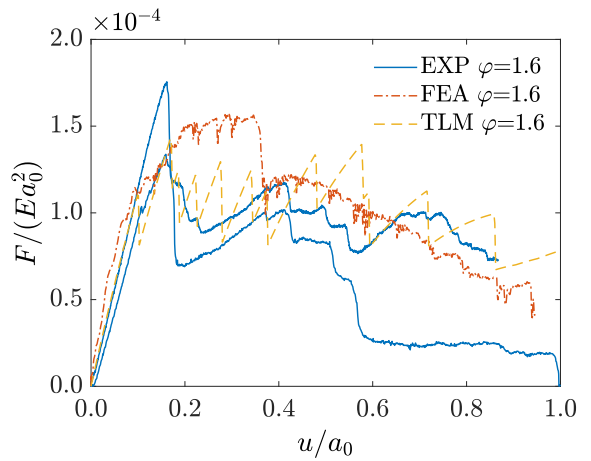

(a)

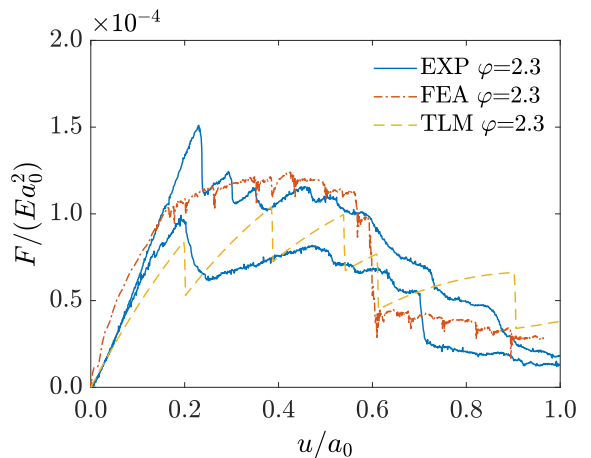

(b)

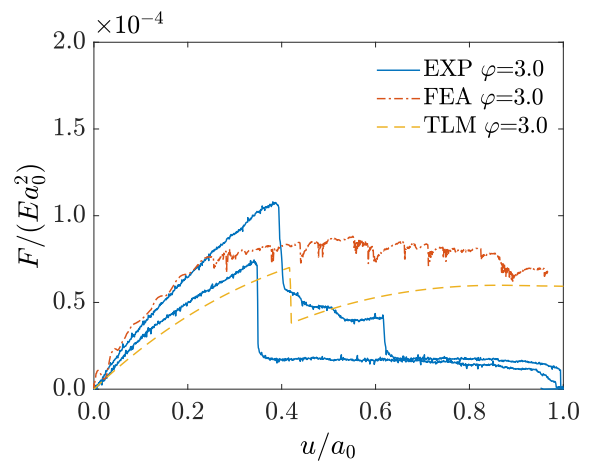

(c)

Figure 14: Comparison of measured TIM tube force-displacement response (EXP) to FEmodel (FEA) and TL-model (TLM) predictions. (a) $\varphi=1.6$, (b) $\varphi=2.3$, and (c) $\varphi=3.0$.

[37]. This entails an arrangement of interlocking building blocks separated by weak interfaces. The TIM tubes require the presence of binding frames. In a planar TIM system, the frame may be a single piece running the perimeter of the assembly. TIM tubes on the other hand require two separate frame parts to constrain the collection of building blocks, which can be fixed to one another such that the system can be handled independently.

Experiments performed on additively manufactured TIM tubes demonstrated that interlocking building blocks result in a stiffer, stronger, and 
tougher configuration than non-interlocking blocks. The failure of noninterlocking tubes $\left(\theta_{\text {int }}=0^{\circ}\right)$ in experiments was solely due to the push-out of the loaded block after the shear failure of surrounding material. On the other hand, the response and failure of TIM tubes $\left(\theta_{\text {int }}=17^{\circ}\right)$ is more complex due to interactions between interlocking building blocks. The sharp decline in load capacity noticed in AM TIM tubes is a result of the failure of the support matrix between building blocks. Before support matrix failure, the TIM building blocks are essentially bonded. This results in the stiffer response before the drop in measured load. After support matrix failure sliding becomes very relevant in the TIM tube response. The soft contacts between building blocks enables the sliding of building blocks relative to one another more easily. As a result the TIM building block located under the flat punch is displaced further relative to neighboring blocks. Interactions in the system caused neighboring blocks to be pushed radially outward, resulting in a less stringent confinement of building blocks. The failure of AM TIM tubes is then a result of cumulative interactions between building blocks that ultimately results in the removal of the loaded block. This is in contrast to the shear-failure push-out observed in non-interlocking tubes, and as such, a theory for the deformation response of TIM tubes is needed.

Numerical simulations of the loading of idealized TIM tubes predict a skew-parabolic load response with no sharp drops in load capacity. The forcedisplacement responses of all TIM tubes displayed softening to some extent. This leads to a more gradual failure than in monolithic brittle materials, and is consistent with previous findings in planar TIM systems [22, 26, 29]. Considering an elastic connection between frames leads to lower stiffness and 
strength predictions, but preserves the shape of the response. Ultimately, this shows that TIM tubes can be realized by fixing frames together with some connection and that the structure's response would be characteristic of TIM systems. The deformation of TIM tubes is a combination of the elastic deformation of building blocks and sliding between building blocks. During the loading of TIM tubes, deformation shifts from mostly elastic deformation to mostly sliding deformation. FE-model energies show that higher aspect ratio TIM tubes are more resistant to sliding. The ultimate failure of each aspect ratio TIM tube occurs after the removal of the center building block.

By modifying the contact conditions in FE-models a TIM tube response like in experiments can be replicated. Scaling down contact stiffness and imposing a frictional shear stress limit results in the lower stiffness and dramatic failure observed in experiments. This is due to the contact modifications allowing increased slip between building blocks. The locomotion of building blocks during deformation also reflects experimental observations. Building blocks other than the loaded one are displaced from their initial positions during the deformation of the TIM tube, which was not observed in the idealized simulations. There is also little energy stored as strain in building blocks, meaning the TIM tube behaves like an collection of rigid bodies under these conditions. This shows that the interaction between blocks is an important factor in the deformation and response of TIM tubes. Weaker interactions between blocks result in a more compliant and lower strength system. This is similar to the findings of [38] where the addition of soft, rubber interfaces between concrete TIM building blocks lowered the TIM system strength.

Analysis of the TIM tube systems through equivalent truss structures 
enabled the force-displacement response to be recreated, like in planar TIM systems. While a network of identical, orthogonal trusses with local displacement criteria was representative of planar TIM systems, crossed trusses of varying span and height were able to capture the stiffer, stronger response of TIM tubes. Previously the stiffness of planar TIM systems were approximated based on material properties, TIM building block size, and building block count. Since trusses were not restricted to spanning the distance between frames while remaining orthogonal to one another, mechanical properties were dependant on a larger set of TIM tube parameters. For the Mises truss, F1, the response stiffness is proportional to the height of the tube. The dimensions of diagonal trusses in the TIM tube, however, are described in terms of tube radius and unit count around the perimeter. Because of this the stiffness of a diagonal truss $F_{i}$ is proportional to a function of material properties, tube radius, building block size, and building block count. Thus, the total stiffness of TIM tubes also includes tube radius in its formulation.

The TL-model provides a good approximation of the stiffness and strength of TIM tubes with larger values of $\varphi$ based on a selection of a parameter value for $E A_{i}$. However, the basic TL-model does not approximate the experimental results well. In both the numerical models and experiments, interfaces between TIM building blocks are not perfectly bonded. The TL-model does not account for sliding, so there exist discrepancies in the failure mechanisms between each. Idealized contact conditions are initially used in numerical models so that sliding between blocks is minimal. As a result the TL-model strength predictions are relatively similar to idealized numerical model results. In experiments on additively manufactured TIM tubes, more sliding 
between building blocks was allowed due to the compliant support matrix. The result is that the TL-model predicts much higher strengths and displacements to failure than that of additively manufactured TIM tubes.

Reformulating the TL-model to account for system deformation in the form of slip yields a load response similar to experimental results. Imposing a critical force cutoff for individual trusses in the model controls the onset of slip and predicted strength of TIM tubes. This is similar to the frictional shear stress cutoff used in the FE-models for the experimental configuration, and may be compared to the failure of support material between blocks in experiments. The global failure mode predicted by the TL-model depends on the fraction of applied displacement allocated to slipping by the slip factor. With a small slip factor the TL-model predicted response displays a gradual failure. This is because only a small amount of applied displacement is accounted for as slip, and the TL-model functions the same otherwise. With larger slip factors more most of the applied displacement is accounted for as slip and noticeable drops in force can be observed. Large slip factors best replicate the large amounts of sliding in additively manufactured TIM tubes after the failure of the support matrix between building blocks.

Either formulation of the TL-model does not work well to predict the response of TIM tubes with $\theta_{\text {int }}=0^{\circ}$. When $\theta_{\text {int }}=0^{\circ}$ there is a considerable amount of slip in assemblies, even at the onset of loading. This is unlike experimental results, where slip is initiated after the strength of the support material is overcome. As a result the thrust-line evolution in TIM tubes with $\theta_{\text {int }}=0^{\circ}$ is unique compared to TIM tubes with $\theta_{\text {int }}=17^{\circ}$. To accurately predict the response of TIM tubes with $\theta_{\text {int }}=0^{\circ}$, the TL-model must be modified 
further to account for this earlier slip between building blocks.

The discrepancies in predicted stiffness between experimental, FE-model, and TL-model results may be a result of the formulation of the TL-model. It is possible that the effective truss area $A_{i}$ varies with tube $\varphi$, between trusses $F_{i}$, or with deflection $u$. The area $A_{i}$ represents the contact area between building blocks within a single force network component. In [39] a deflectiondependent contact area is used in a model for Voussier beams. The rotation of beam segments is considered to update the contact area used in analysis. In the case of TIM tubes a similar method could be applied to account for the rotation and sliding of building blocks. The present thrust-line TIM tube model does not account changes in contact area. $A_{i}$ was assumed constant for all trusses. Also, truss abutments were restricted to have fixed abutments located on the TIM tube's bounding frames. Each TIM unit within the frame is also limited to having one truss abutment. No more diagonal frame-toframe trusses can be incorporated into the model without thrust-lines leaving the tube volume, so TIM tube response formulation may include trusses with smaller spans and dynamic abutments somewhere within the plurality of TIM building blocks. The implication of this is that load transfer in a TIM tube is more complex than previously seen planar TIM systems. In addition to equivalent trusses now being non-orthogonal, it seems that fixed abutment trusses spanning frame-to-frame yield an incomplete system response.

Only a handful of possible TIM tube configurations were considered in this study - all of which were crafted from square tessellations. It is possible to create TIM tubes with other tessellation patterns, and the result of doing so is unknown. Additionally, only tube height was altered in this 
study, with tube radius, tessellation size, and interlocking angle being held constant. Modifying these other parameters would certainly influence TIM tube response, and this should be kept in consideration.

\section{Conclusion}

TIM tubes composed of repeated quasi-tetrahedral units were considered as a novel topologically interlocking material system. The present work demonstrated the significant increase in strength and toughness that can be achieved by interlocking geometries relative to non-interlocking geometries. Mechanisms in the realizations of strong and tough biologically-inspired structures were explained with fundamental mechanics of materials concepts. The deformation and load response of TIM tubes with multiple boundary conditions were closely monitored in FE computations. TIM tube response was found to exhibit a gradual failure in computations, like previously demonstrated in planar TIM systems. The strength and failure of TIM tubes was found to depend on the the dimensions of the tube as well as the contact conditions between building blocks. The response of TIM tubes was found to be a product of the formation of force networks. Force network geometry was used to describe equivalent truss structures in a thrust-line model of the system. The formulated thrust-line model was able to approximate the stiffness of the computed and experimental TIM tube load response. Modifying the model to include slip between building blocks resulted in a better approximation of TIM tube strength and failure under experimental conditions. This model contains a new parameter which is not present in planar TIM models (tube radius), so the mechanical response can be further modulated with this 
parameter.

Acknowledgment: This work is supported by the National Science Foundation under Grant No. 1662177. 


\section{Appendix A. Mesh Convergence}

To determine an appropriate mesh density for TIM building blocks, results using a range of mesh seeds per side is considered. Mesh densities of 5 to 20 seeds per building block edge are considered. The geometry used in this convergence study was that of the TIM tube with $\varphi=3.0$. The TIM tube considered was subjected to loading by flat punch indentation and a fixed end boundary condition. The computed value of the computed maximum reaction force, $F_{\max }$, is used to determine an investigate mesh density. Values of $F_{\max }$ in dependence of mesh density are shown in Fig. A.15a. $F_{\max }$ is normalized with by the material elastic modulus multiplied by the building block cross-section area. A mesh density of 15 seeds per side was selected as $F_{\max }$ values do not vary by more than $1 \%$ with higher mesh density. It was also found that certain mesh densities cause a large viscous dissipation contribution in the computed solution. The reason for the presence of vis-

cous dissipation is to resolve contact issues between edges and corners of some elements. Figure A.15b shows the ratio of viscous dissipation to total applied work in models with various mesh densities. It was decided that the viscous dissipation contribution is acceptable at the selected mesh density of 15 seeds per side. 


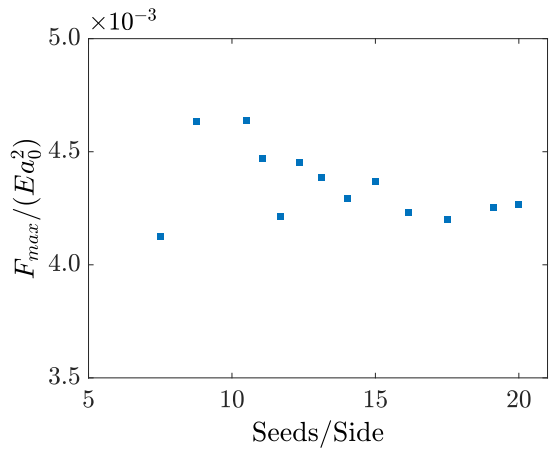

(a)

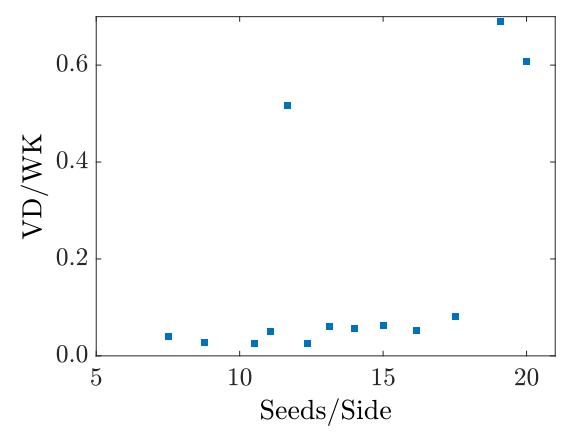

(b)

Figure A.15: (a) Maximum computed force relative to mesh density and (b) ratio of viscous dissipation energy (VD) to total applied work (WK) relative to mesh density.

\section{References}

[1] M. F. Ashby, Hybrids to fill holes in material property space, Philosophical Magazine 85 (2005) 3235-3257. doi:10.1080/14786430500079892.

[2] M. Ashby, Hybrid materials to expand the boundaries of materialproperty space, Journal of the American Ceramic Society 94 (2011) s3-s14. doi:https://doi.org/10.1111/j.1551-2916.2011.04559.x.

[3] N. A. Fleck, V. S. Deshpande, M. F. Ashby, Micro-architectured materials: past, present and future, Proceedings of the Royal Society A: Mathematical, Physical and Engineering Sciences 466 (2010) 2495-2516. doi:10.1098/rspa.2010.0215.

[4] S. Li, H. Fang, S. Sadeghi, P. Bhovad, K.-W. Wang, Architected origami materials: How folding creates sophisticated mechanical properties, Advanced Materials 31 (2019) 1805282. doi:https://doi.org/10.1002/adma.201805282. 
[5] A. Dyskin, Y. Estrin, A. Kanel-Belov, E. Pasternak, A new concept in design of materials and structures: assemblies of interlocked tetrahedron-shaped elements, Scripta Materialia 44 (2001) 2689-2694. doi:10.1016/S1359-6462(01)00968-X.

[6] F. Barthelat, Architectured materials in engineering and biology: fabrication, structure, mechanics and performance, International Materials Reviews 60 (2015) 413-430. doi:10.1179/1743280415Y.0000000008.

[7] D. Zhu, C. F. Ortega, R. Motamedi, L. Szewciw, F. Vernerey, F. Barthelat, Structure and mechanical performance of a "modern" fish scale, Advanced Engineering Materials 14 (2012) B185-B194. doi:https://doi.org/10.1002/adem.201180057.

[8] S. Levin, The tensegrity-truss as a model for spine mechanics: Biotensegrity, Journal of Mechanics in Medicine and Biology 02 (2011). doi:10.1142/S0219519402000472.

[9] B. Achrai, H. D. Wagner, The turtle carapace as an optimized multi-scale biological composite armor - A review, Journal of the Mechanical Behavior of Biomedical Materials 73 (2017) 50-67. doi:10.1016/j.jmbbm.2017.02.027.

[10] N. S. Ha, G. Lu, A review of recent research on bio-inspired structures and materials for energy absorption applications, Composites Part B: Engineering 181 (2020) 107496. doi:10.1016/j.compositesb.2019.107496.

[11] J. C. Huss, S. J. Antreich, J. Bachmayr, N. Xiao, M. Eder, J. Konnerth, N. Gierlinger, Topological interlocking and geometric stiffening as com- 
plementary strategies for strong plant shells, Advanced Materials 32 (2020) 2004519. doi:https://doi.org/10.1002/adma.202004519.

[12] G. Blanchard, A 3D cell shape that enables tube formation, Nature 561 (2018) 182-183. doi:10.1038/d41586-018-06162-1.

[13] K. Moreno Gata, C. Mueller, E. Valiente, Designing strategies for topological interlocking assemblies in architecture. Flat Vaults, 2019.

[14] P. Block, T. Ciblac, J. Ochsendorf, Real-time limit analysis of vaulted masonry buildings, Computers \& Structures 84 (2006) 1841-1852. doi:10.1016/j.compstruc.2006.08.002.

[15] O. Tessmann, Topological interlocking assemblies, Digital Physicality: Proceedings of ECAADe 2012 (2012) 211-219.

[16] G. Fallacara, S. Macro, Stereotomy modern stone architecture and its historical legacy, 2012.

[17] M. Brocato, W. Deleporte, L. Mondardini, J.-E. Tanguy, A Proposal for a New Type of Prefabricated Stone Wall, International Journal of Space Structures 29 (2014) 97-112. doi:10.1260/0266-3511.29.2.97.

[18] V. Loing, O. Baverel, J.-F. Caron, R. Mesnil, Free-form structures from topologically interlocking masonries, Automation in Construction 113 (2020) 103117. doi:10.1016/j.autcon.2020.103117.

[19] M. Weizmann, O. Amir, J. Grobman, Topological interlocking in architectural design, 2015. doi:10.13140/RG.2.1.3642.3766. 
[20] M. Weizmann, O. Amir, J. Grobman, Topological interlocking in buildings: A case for the design and construction of floors, Automation in Construction 72 (2016). doi:10.1016/j.autcon.2016.05.014.

[21] Y. Estrin, A. V. Dyskin, E. Pasternak, Topological interlocking as a material design concept, Materials Science and Engineering: C 31 (2011) 1189-1194. doi:10.1016/j.msec.2010.11.011.

[22] S. Khandelwal, T. Siegmund, R. J. Cipra, J. S. Bolton, Transverse loading of cellular topologically interlocked materials, International Journal of Solids and Structures 49 (2012) 2394-2403. doi:10.1016/j.ijsolstr.2012.04.035.

[23] T. Siegmund, F. Barthelat, R. Cipra, E. Habtour, J. Riddick, Manufacture and mechanics of topologically interlocked material assemblies, Applied Mechanics Reviews 68 (2016). doi:10.1115/1.4033967.

[24] A. Dyskin, Y. Estrin, E. Pasternak, Topological Interlocking Materials, in: Springer Series in Materials Science, 2019, pp. 23-49. doi:10.1007/978-3-030-11942-3-2.

[25] M. Mirkhalaf, T. Zhou, F. Barthelat, Simultaneous improvements of strength and toughness in topologically interlocked ceramics, Proceedings of the National Academy of Sciences 115 (2018) 9128-9133. doi:10.1073/pnas.1807272115.

[26] A. Williams, T. Siegmund, Mechanics of topologically interlocked material systems under point load: Archimedean and Laves tiling, 
International Journal of Mechanical Sciences 190 (2021) 106016. doi:10.1016/j.ijmecsci.2020.106016.

[27] M. Weizmann, O. Amir, Y. J. Grobman, The effect of block geometry on structural behavior of topological interlocking assemblies, Automation in Construction 128 (2021) 103717. doi:10.1016/j.autcon.2021.103717.

[28] S. Khandelwal, T. Siegmund, R. J. Cipra, J. S. Bolton, Scaling of the elastic behavior of two-dimensional topologically interlocked materials under transverse loading, Journal of Applied Mechanics 81 (2014). doi:10.1115/1.4024907.

[29] M. Short, T. Siegmund, Scaling, growth, and size effects on the mechanical behavior of a topologically interlocking material based on tetrahedra elements, Journal of Applied Mechanics 86 (2019). doi:10.1115/1.4044025, publisher: American Society of Mechanical Engineers Digital Collection.

[30] M. Brocato, L. Mondardini, A new type of stone dome based on Abeille's bond, International Journal of Solids and Structures 49 (2012) 17861801. doi:10.1016/j.ijsolstr.2012.03.036.

[31] Z. Wang, P. Song, F. Isvoranu, M. Pauly, Design and structural optimization of topological interlocking assemblies, ACM Transactions on Graphics 38 (2019) 1-13. doi:10.1145/3355089.3356489.

[32] A. Bejarano, C. Hoffmann, A generalized framework for designing topological interlocking configurations, International Journal of Architectural Computing 17 (2019) 53-73. doi:10.1177/1478077119827187. 
[33] W. Xu, X. Lin, Y. M. Xie, A novel non-planar interlocking element for tubular structures, Tunnelling and Underground Space Technology 103 (2020) 103503. doi:10.1016/j.tust.2020.103503.

[34] H.-C. Ries, M. Vieira Carlesso, C. Eigenbrod, S. Kroll, K. Rezwan, On the performance of porous sound absorbent ceramic lining in a combustion chamber test rig, volume 4, 2013. doi:10.1115/GT2013-95492.

[35] N. Suksangpanya, N. A. Yaraghi, R. B. Pipes, D. Kisailus, P. Zavattieri, Crack twisting and toughening strategies in Bouligand architectures, International Journal of Solids and Structures 150 (2018) 83-106. doi:10.1016/j.ijsolstr.2018.06.004.

[36] J. Q. Ran, E. K. S. Passaris, P. Mottahed, Shear sliding failure of the jointed roof in laminated rock mass, Rock Mechanics and Rock Engineering 27 (1994) 235-251. doi:10.1007/BF01020201.

[37] M. Mirkhalaf, J. Tanguay, F. Barthelat, Carving 3D architectures within glass: Exploring new strategies to transform the mechanics and performance of materials, Extreme Mechanics Letters 7 (2016) 104-113. doi:10.1016/j.eml.2016.02.016.

[38] A. Rezaee Javan, H. Seifi, X. Lin, Y. M. Xie, Mechanical behaviour of composite structures made of topologically interlocking concrete bricks with soft interfaces, Materials \& Design 186 (2020) 108347. doi:10.1016/j.matdes.2019.108347.

[39] M. S. Diederichs, P. K. Kaiser, Stability of large excavations in laminated hard rock masses: the voussoir analogue revisited, Interna- 
tional Journal of Rock Mechanics and Mining Sciences 36 (1999) 97-117. doi:10.1016/S0148-9062(98)00180-6. 